\title{
COHOMOLOGY THEORIES ON SPACES
}

\author{
E. SPANIER
}

\begin{abstract}
In this paper a previously proven uniqueness theorem for nonnegative cohomology theories on the same space is extended to cohomology theories on the same finite-dimensional space. In this form it is applicable to extraordinary cohomology theories. An example is given to show that the theorem does not hold without finite dimensionality.
\end{abstract}

1. Introduction. This paper is a study of cohomology theories of various types on a topological space. For our purposes a cohomology theory on a space $X$ is a continuous exact contravariant functor from the category of closed subsets of $X$ to the category of graded abelian groups. A homomorphism between two cohomology theories on the same space is a natural transformation between the contravariant functors which maps the exact sequences of one into the exact sequences of the other. We are interested in uniqueness theorems asserting that a homomorphism which is an isomorphism for every point of the space as an isomorphism for every closed subset of the space. Such theorems were proved in [5, 9] for nonnegative cohomology theories. These theorems have as consequences many results in ordinary cohomology theory previously obtained using sheaf theory.

In the present paper the condition of nonnegativity is dropped so that the theory may be applied to extraordinary cohomology theories. In this case the uniqueness theorem is not valid without some additional assumption. We prove it is valid for finite-dimensional spaces. This form of the uniqueness theorem has some interesting applications to cohomology of manifolds. It can also be applied to prove a similar uniqueness theorem for cohomology theories defined on larger categories such as the category of all compact spaces and continuous functions or the category of all locally compact spaces and proper continuous functions.

The rest of the paper is divided into three sections. $\$ 2$ contains the definition of cohomology theory on a space and some of its elementary properties. $\$ 3$ contains a proof that the cohomology theories considered by Lawson [5] are essentially the same as ours and that ES theories (which satisfy Eilenberg-Steenrod axioms) determine cohomology theories. $\$ 4$ is devoted to a proof of the uniqueness theorem for finite-dimensional spaces and to applications of this result to manifolds.

Received by the editors April 14, 1986 and, in revised form June 4, 1986.

1980 Mathematics Subject Classification (1985 Revision). Primary 55N40; Secondary 55N20, 55N30.

The author gratefully acknowledges partial support and/or hospitality by the Forschungsinstitut für Mathematik, E. T. H., Zürich, the Institute des Hautes Etudes Scientifiques, Bures-sur-Yvette, and the Max-Planck-Institut für Mathematik, Bonn, during the preparation of this paper. 
2. Cohomology on a space. In this section we present the definition of a cohomology theory on a space $X$ and some elementary properties of such theories. The present definition differs from that in [9] in that nonnegativity is not assumed.

All topological spaces will be assumed to be normal Hausdorff spaces. If $H$ is a contravariant functor from a category of subsets (and inclusion maps between them) of such a space to the category of graded abelian groups (and homomorphisms of degree zero between them), we use the following notation. If $H$ is defined for an inclusion map $i: B \subset A$ and $u \in H(A)$, then $u \mid B \in H(B)$ is defined by $u \mid B=$ $H(i)(u)$. With this notation the statement that $H$ is a contravariant functor is equivalent to the two conditions:

(i) for $u \in H(A), u \mid A=u$, and

(ii) for $C \subset B \subset A$ and $u \in H(A)$ then $(u \mid B)|C=u| C$.

In general we will use $\rho$ to denote a homomorphism induced by an inclusion map (i.e. $\rho=H(i): H(A) \rightarrow H(B)$ for $i: B \subset A$ ). Given a topological space $X$ let $\operatorname{cl}(X)$ denote the category of all closed subsets of $X$ and all inclusion maps between them. A cohomology theory $H, \delta$ on $X$ consists of (i) a contravariant functor $H$ from $\operatorname{cl}(X)$ to the category of graded abelian groups $\left(H(X)=\left\{H^{q}(X)\right\}_{q \in \mathbf{Z}}\right)$ such that $H(\varnothing)$ $=0$ and (ii) a natural transformation $\delta$ assigning to every two closed subsets $A, B \subset X$ a homomorphism of degree 1, $\delta: H(A \cap B) \rightarrow H(A \cup B)$, such that the following are satisfied.

Continuity. For every closed $A \subset X$ there is an isomorphism

$$
\rho: \underset{\lim }{\rightarrow}\left\{H^{q}(N) \mid N \text { a closed neighborhood of } A \text { in } X\right\} \approx H^{q}(A)
$$

where $\rho\{u\}=u \mid A$ for $u \in H^{q}(N)$.

MV ExACTNESS. For every two closed sets $A, B$ in $X$ there is an exact sequence

$$
\cdots \stackrel{\delta}{\rightarrow} H^{q}(A \cup B) \stackrel{\alpha}{\rightarrow} H^{q}(A) \oplus H^{q}(B) \stackrel{\beta}{\rightarrow} H^{q}(A \cap B) \stackrel{\delta}{\rightarrow} H^{q+1}(A \cup B) \stackrel{\alpha}{\rightarrow} \cdots
$$

where $\alpha(u)=(u|A, u| B)$ for $u \in H^{q}(A \cup B)$ and $\beta(u, v)=u \mid A \cap B-$ $v \mid A \cap B$ for $u \in H^{q}(A), v \in H^{q}(B)$.

REMARKS. (2.1) This definition differs from that in [9] in that it has not been assumed that $H^{q}(A)=0$ for $q<0$ for all closed $A \subset X$. This is a substantial difference in that the uniqueness theorem [9, Theorem 2.20] is only proved for finite-dimensional spaces $X$ and, in fact, is false for arbitrary compact spaces (see $\S 4$ below).

(2.2) Note that the continuity property does not involve the natural transformation $\delta$. Thus, we speak of a continuous $H$ and an exact $H, \delta$.

(2.3) If $H, \delta$ is a cohomology theory on $X$, then its $p$ th suspension $\sigma^{p} H, \delta$ where $\left(\sigma^{p} H\right)^{q}(A)=H^{p+q}(A)$ and $\delta: \quad H^{p+q}(A \cap B) \rightarrow H^{p+q+1}(A \cup B)$ is also a cohomology theory on $X$ for every $p \in \mathbf{Z}$.

(2.4) If $\left\{H_{j}, \delta_{j}\right\}_{j \in J}$ is a family of cohomology theories on $X$ indexed by an arbitrary set $J$, then the direct sum $\oplus_{j \in J} H_{j}, \oplus_{j \in J} \delta_{j}$ where

and

$$
\left(\bigoplus_{j \in J} H_{j}\right)^{q}(A)=\bigoplus_{j \in J} H_{j}^{q}(A)
$$

$$
\bigoplus_{j \in J} \delta_{j}: \bigoplus_{j \in J} H_{j}^{q}(A \cap B) \rightarrow \bigoplus_{j \in J} H_{j}^{q+1}(A \cup B)
$$


is also a cohomology theory on $X$ (because direct sums commute with direct limits and preserve exactness).

(2.5) If $H, \delta$ is a cohomology theory on $X$ and $G$ is a torsion-free abelian group the tensor product $H \otimes G, \delta \otimes 1_{G}$ where $(H \otimes G)^{q}(A)=H^{q}(A) \otimes G$ and $\delta \otimes 1_{G}$ : $H^{q}(A \cap B) \otimes G \rightarrow H^{q+1}(A \cup B) \cap G$ is also a cohomology theory on $X$ (because tensor product with $G$ commutes with direct limits and, because $G$ is torsion-free, preserves exactness).

(2.6) Let $f: X \rightarrow Y$ be a closed continuous map and let $H, \delta$ be a cohomology theory on $X$. The direct image $f_{*} H, \delta$ where $\left(f_{*} H\right)^{q}(A)=H^{q}\left(f^{-1}(A)\right)$ for $A \subset Y$ and $\delta: H^{q}\left(f^{-1}(A) \cap f^{-1}(B)\right) \rightarrow H^{q+1}\left(f^{-1}(A) \cup f^{-1}(B)\right)$ is a cohomology theory on $Y$ (closedness of $f$ and normality of $Y$ imply that the collection $\left\{f^{-1}(N) \mid N\right.$ a closed neighborhood of $A$ in $Y\}$ is cofinal in the collection of closed neighborhoods of $f^{-1}(A)$ in $\left.X\right)$.

The following proposition concerns two consequences of the continuity property of cohomology theories.

Proposition (2.7). Let $H$ be a continuous contravariant functor on $\mathrm{cl}(X)$.

(1) If $A \subset A^{\prime}$ are closed subsets of $X$, there is an isomorphism

$$
\rho: \lim _{\rightarrow}\left\{H^{q}(N) \mid N \text { a closed neighborhood of } A \text { in } A^{\prime}\right\} \approx H^{q}(A)
$$

where $\rho\{u\}=u \mid A$ for $u \in H^{q}(N)$.

(2) If $\left\{A_{j}\right\}_{j \in J}$ is a family of compact subsets of $X$ directed downward by inclusion, there is an isomorphism

$$
\rho: \lim _{\rightarrow}\left\{H^{q}\left(A_{j}\right)\right\}_{j \in J} \approx H^{q}\left(\bigcap_{j \in J} A_{j}\right)
$$

where $\rho\{u\}=u \mid \cap_{j \in J} A_{j}$ for $u \in H^{q}\left(A_{j}\right)$.

Proof. (1) follows as did the corresponding property for the cohomology theories previously defined [9, Lemma 2.13]. (2) follows from continuity using the fact that compactness of $A_{j}$ for each $j \in J$ implies that if $N$ is any neighborhood of $\bigcap_{j \in J} A_{j}$ there is $j \in J$ such that $A_{j} \subset N$.

A cohomology theory $H, \delta$ is said to be nonnegative if $H^{q}(A)=0$ for $q<0$ and all closed $A \subset X$. The cohomology theories considered in [9] were nonnegative cohomology theories.

A cohomology theory $H, \delta$ on $X$ is said to be compactly supported (or to have compact supports) if given $u \in H^{q}(A)$ there is a decomposition $A=B \cup C$ where $B$ is closed, $C$ is compact, and $u \mid B=0$.

A cohomology theory $H, \delta$ is said to be additive if given a discrete ${ }^{1}$ family $\left\{A_{j}\right\}_{j \in J}$ of closed sets, there is an isomorphism $H^{q}\left(\cup_{j \in J} A_{j}\right) \approx \prod_{j \in J} H^{q}\left(A_{j}\right)$ sending $u \in H^{q}\left(\bigcup_{j \in J} A_{j}\right)$ to the family $\left\{u \mid A_{j}\right\}_{j \in J}$.

\footnotetext{
${ }^{1} \mathrm{~A}$ family $\left\{A_{j}\right\}_{j \in J}$ of subsets of a topological space is said to be discrete if every point of $X$ has a neighborhood meeting at most one member of the family. This implies the members of the family are pairwise disjoint and, since a discrete family is obviously locally finite, if each is closed in $X$, then $\bigcup_{j \in J} A_{j}$ is also closed in $X$.
} 
In none of the above definitions does the natural transformation $\delta$ enter.

If $H, \delta$ and $H^{\prime}, \delta^{\prime}$ are cohomology theories on the same space $X$, a homomorphism $\phi$ from $H, \delta$ to $H^{\prime}, \delta^{\prime}$ is a natural transformation from $H$ to $H^{\prime}$ commuting up to sign with $\delta, \delta^{\prime}$.

Recall [9] that a homomorphism $\phi: G \rightarrow G^{\prime}$ of degree 0 between graded abelian groups is an n-equivalence if $\phi: G^{q} \rightarrow G^{\prime q}$ is an isomorphism for all $q<n$ and a monomorphism for $q=n$. We prove two results about homomorphisms between cohomology theories on the same space $X$ which are assumed to be $n$-equivalences for certain subsets of $X$. (Actually neither result requires the continuity property.)

Proposition (2.8). Let $\phi: H, \delta \rightarrow H^{\prime}, \delta^{\prime}$ be a homomorphism between two compactly supported cohomology theories on $X$ and suppose there is $n \in \mathbf{Z}$ such that $\phi_{C}$ : $H(C) \rightarrow H^{\prime}(C)$ is an n-equivalence for every compact $C \subset X$. Then $\phi_{A}: H(A) \rightarrow$ $H^{\prime}(A)$ is an n-equivalence for every closed $A \subset X$.

Proof. (1) We prove $\phi_{A}: H^{q}(A) \rightarrow H^{\prime q}(A)$ is an epimorphism for $q<n$ and $A$ closed in $X$. Let $u \in H^{\prime q}(A)$ where $q<n$. Since $H^{\prime}$ is compactly supported, $A=B \cup C$ where $B$ is closed, $C$ is compact, and $u \mid B=0$. The following diagram has exact rows and commutes up to sign

$$
\begin{array}{ccccccc}
H^{q-1}(B \cap C) & \stackrel{\delta}{\rightarrow} & H^{q}(A) & \stackrel{\alpha}{\rightarrow} & H^{q}(B) \oplus H^{q}(C) & \stackrel{\beta}{\rightarrow} & H^{q}(B \cap C) \\
\phi \downarrow \approx & & \phi \downarrow & & \downarrow \phi & & \approx \downarrow \phi \\
H^{\prime q-1}(B \cap C) & \stackrel{\delta^{\prime}}{\rightarrow} & H^{\prime q}(A) & \stackrel{\alpha^{\prime}}{\rightarrow} & H^{\prime q}(B) \oplus H^{\prime q}(C) & \stackrel{\beta^{\prime}}{\rightarrow} & H^{\prime q}(B \cap C)
\end{array}
$$

and the vertical maps on the two ends are isomorphisms because $B \cap C$ is compact and $q<n$. By [9, Lemma 2.19, part 2], $\alpha^{-1}(\operatorname{im} \phi) \subset \operatorname{im} \phi$. Since $\phi_{C}: H^{q}(C) \approx$ $H^{\prime q}(C)$ there is $v \in H^{q}(C)$ such that $\phi(v)=u \mid C$. Then $(0, v) \in H^{q}(B) \oplus H^{q}(C)$ is such that $\phi(0, v)=(0, \phi(v))=(0, u \mid C)=\alpha^{\prime}(u)$ so $\alpha^{\prime}(u) \in \operatorname{im} \phi$ and, hence, $u \in$ $\operatorname{im} \phi$.

(2) We prove $\phi_{A}: H^{q}(A) \rightarrow H^{\prime q}(A)$ is a monomorphism for $q \leqslant n$. Assume $u \in H^{q}(A), q \leqslant n$ is such that $\phi(u)=0$. Because $H$ has compact supports, $A=$ $B \cup C$ where $B$ is closed, $C$ is compact, and $u \mid B=0$. The following diagram has exact rows and commutes up to sign

$$
\begin{array}{cccccc}
H^{q-1}(B) \oplus H^{q-1}(C) & \stackrel{\beta}{\rightarrow} H^{q-1}(B \cap C) & \stackrel{\delta}{\rightarrow} H^{q}(A) & \stackrel{\alpha}{\rightarrow} H^{q}(B) \oplus H^{q}(C) \\
\phi \downarrow & & \phi \downarrow \approx & \downarrow \phi & \downarrow \phi \\
H^{\prime q-1}(B) \oplus H^{\prime q-1}(C) & \stackrel{\beta^{\prime}}{\rightarrow} H^{\prime q-1}(B \cap C) & \stackrel{\delta^{\prime}}{\rightarrow} & H^{\prime q}(A) & \stackrel{\alpha^{\prime}}{\rightarrow} & H^{\prime q}(B) \oplus H^{\prime q}(C)
\end{array}
$$

and the first vertical map is an epimorphism by (1) above and the second vertical map is an isomorphism because $B \cap C$ is compact and $q-1<n$. By [9, Lemma 2.19, part 1] $\operatorname{ker} \alpha \cap \operatorname{ker} \phi=0$. Since $\phi_{C}: H^{q}(C) \rightarrow H^{\prime q}(C)$ is a monomorphism for $q \leqslant n$ it follows that $u \mid C=0($ since $\phi(u)=0$ ). Therefore, $\alpha(u)=0$ so $u \in \operatorname{ker} \phi \cap$ ker $\alpha$ and so $u=0$. 
Proposition (2.9). Let $\phi: H, \delta \rightarrow H^{\prime}, \delta^{\prime}$ be a homomorphism between two additive cohomology theories on a paracompact space $X$. Suppose there is $n \in \mathbf{Z}$ and an open covering $\mathscr{U}$ of $X$ such that $\phi_{A}: H(A) \rightarrow H^{\prime}(A)$ is an n-equivalence for every $A$ contained in some element of $\mathscr{U}$. Then $\phi_{A}: H(A) \rightarrow H^{\prime}(A)$ is an n-equivalence for every closed $A \subset X$.

Proof. Let $\mathscr{C}$ be the collection of closed subsets $A$ of $X$ such that $\phi_{B}$ : $H(B) \rightarrow H^{\prime}(B)$ is an $n$-equivalence for every closed $B \subset A$. By the hypothesis of the Proposition every point of $X$ has a closed neighborhood in $\mathscr{C}$. From the definition of $\mathscr{C}$ it is clear that if $A^{\prime}, A$ are closed sets with $A^{\prime} \subset A$ and $A \in \mathscr{C}$, then $A^{\prime} \in \mathscr{C}$. It follows from the exactness of $H, \delta$ and of $H^{\prime}, \delta^{\prime}$ that if $A, B$ are in $\mathscr{C}$ then $A \cup B \in \mathscr{C}$. It follows from the additivity of $H, \delta$ and $H^{\prime}, \delta^{\prime}$ that if $\left\{A_{j}\right\}_{j \in J}$ is a discrete family in $\mathscr{C}$, then $\bigcup_{j \in J} A_{j}$ is also in $\mathscr{C}$. Hence, $\mathscr{C}$ satisfies the hypotheses of [7, Theorem 5.5] so $X \in \mathscr{C}$.

3. Other theories. In [5] Lawson considered cohomology theories satisfying axioms similar to, but somewhat different from, those defined in $\$ 2$. We prove that his definition is essentially equivalent to ours. We also consider theories defined on $\operatorname{cl}(X)^{2}$ satisfying axioms similar to those of Eilenberg and Steenrod [2] and show that they define cohomology theories.

An $L$ theory $H, \Delta$ on $X$ is defined to be a pointwise taut cohomology theory on $X$ in the sense of [5]. Thus, it consists of:

(i) A contravariant functor $H$ from $\operatorname{cl}(X)$ to nonnegative graded abelian groups such that $H(\varnothing)=0$, and

(ii) A natural transformation of degree 1, $\Delta: H^{q}(A \cap B) \rightarrow H^{q+1}(A \cup B)$ defined for every two closed subsets $A, B$ of $X$ such that $\operatorname{int}_{A \cup B} A \cup$ int $_{A \cup B} B=$ $A \cup B$ (in which case we say $A, B$ are an excisive couple in $X$ ), satisfying

(1) For every $x \in A$ where $A$ is closed in $X$ there is an isomorphism

$$
\rho: \lim _{\rightarrow}\left\{H^{q}(N) \mid N \text { a closed neighborhood of } x \text { in } A\right\} \approx H^{q}(x)
$$

such that $\rho\{u\}=u \mid x$ for $u \in H^{q}(N)$.

(2) For every excisive couple $A, B$ in $X$ there is an exact sequence

$$
\ldots \stackrel{\Delta}{\rightarrow} H^{q}(A \cup B) \stackrel{\alpha}{\rightarrow} H^{q}(A) \oplus H^{q}(B) \stackrel{\beta}{\rightarrow} H^{q}(A \cap B) \stackrel{\Delta}{\rightarrow} H^{q+1}(A \cup B) \stackrel{\alpha}{\rightarrow} \ldots
$$

where $\alpha(u)=(u|A, u| B)$ and $\beta(u, v)=u|A \cap B-v| A \cap B$.

Thus, an $L$ theory differs from a cohomology theory in two respects. Firstly, in an $L$ theory the continuity property (1) is for a point $x$ in a closed set $A$ rather than for a closed set $A$ in $X$. Secondly, in an $L$ theory the natural transformation $\Delta$ is defined and the exactness property (2) of $H, \Delta$ is required only for excisive couples in $X$ whereas in a cohomology theory $\delta$ is defined and exactness of $H, \delta$ is required for every two closed sets in $X$.

An $L$ theory is additive if it satisfies the same additivity property defined in $\$ 2$ for cohomology theories. The following shows that on paracompact spaces additive $L$ theories are essentially the same as additive nonnegative cohomology theories. 
THEOREM (3.1). Every nonnegative cohomology theory on $X$ is an L theory on $X$. On a paracompact space $X$ every additive $L$ theory can be extended to an additive nonnegative cohomology theory on $X$.

Proof. If $H, \delta$ is a nonnegative cohomology theory on $X$, then (1) above follows from (1) of Proposition (2.7) and (2) above is $M V$ exactness for excisive couples in $X$ which is a consequence of $M V$ exactness for every two closed sets $A, B$ in $X$. Thus, $H, \delta$ is an $L$ theory on $X$.

Conversely, given $H, \Delta$ an additive $L$ theory on $X$, we show how to extend $\Delta$ to a natural transformation $\delta$ defined for every two closed sets in $X$. Given $A$ closed in $X$ define

$$
\bar{H}^{q}(A)=\lim _{\rightarrow}\left\{H^{q}(N) \mid N \text { a closed neighborhood of } A \text { in } X\right\} .
$$

Then $\bar{H}$ is obviously a contravariant functor from $\operatorname{cl}(X)$ to nonnegative graded abelian groups such that $\bar{H}(\varnothing)=0$ and satisfying the continuity property.

To define a natural transformation of degree $1, \bar{\delta}: \bar{H}^{q}(A \cap B) \rightarrow H^{q+1}(A \cup B)$ such that $\bar{H}, \bar{\delta}$ satisfy $M V$ exactness for every two closed sets $A, B \subset X$ note that because $X$ is normal, it is easy to verify that if $A, B$ are closed sets in $X$ and $U, V$, $W$ are open neighborhoods of $A, B, A \cap B$, respectively, in $X$, there exist closed neighborhoods $M, N$ of $A, B$, respectively, such that $M, N$ is an excisive couple and $M \subset U, N \subset V, M \cap N \subset W$.

Thus, as $M, N$ vary over closed neighborhoods of $A, B$, respectively, such that $M, N$ is an excisive couple in $X$, it follows that $M, N, M \cap N, M \cup N$ vary over a cofinal family of closed neighborhoods of $A, B, A \cap B, A \cup B$, respectively. The direct limit of the exact sequences

$$
\ldots \stackrel{\Delta}{\rightarrow} H^{q}(M \cup N) \stackrel{\alpha}{\rightarrow} H^{q}(M) \oplus H^{q}(N) \stackrel{\beta}{\rightarrow} H^{q}(M \cap N) \stackrel{\Delta}{\rightarrow} H^{q+1}(M \cup N) \rightarrow \cdots
$$

is an exact sequence

$$
\cdots \stackrel{\bar{\delta}}{\rightarrow} \bar{H}^{q}(A \cup B) \stackrel{\bar{\alpha}}{\rightarrow} \bar{H}^{q}(A) \oplus \bar{H}^{q}(B) \stackrel{\bar{\beta}}{\rightarrow} \bar{H}^{q}(A \cap B) \stackrel{\bar{\delta}}{\rightarrow} \bar{H}^{q+1}(A \cup B) \rightarrow \cdots
$$

and defines the natural transformation $\bar{\delta}: \bar{H}^{q}(A \cap B) \rightarrow \bar{H}^{q+1}(A \cup B)$.

Therefore, $\bar{H}, \bar{\delta}$ is a nonnegative cohomology theory on $X$. We show it is additive. Let $\left\{A_{j}\right\}_{j \in J}$ be a discrete family of closed subsets of $X$. For each $j^{\prime}$ let $U_{j^{\prime}}=X-$ $\bigcup_{j \neq j^{\prime}} A_{j}$. Then $\mathscr{U}=\left\{U_{j^{\prime}}\right\}_{j^{\prime} \in J}$ is an open covering of $X$ by sets each of which meets at most one member of $\left\{A_{j}\right\}$. Let $\mathscr{V}$ be an open star refinement of $\mathscr{U}$ (which exists because $X$ is paracompact [1]) and, for each $j \in J$, let

$$
V_{j}=\bigcup\left\{V \in \mathscr{V} \mid V \cap A_{j} \neq \varnothing\right\} .
$$

Then $A_{j} \subset V_{j} \subset U_{j}$. Furthermore, if $V$ is any element of $\mathscr{V}$ and $V \cap V_{j} \neq \varnothing$, $V \cap V_{k} \neq \varnothing$ for $j, k \in J$ there are $V^{\prime}, V^{\prime \prime} \in \mathscr{V}$ with $V \cap V^{\prime} \neq \varnothing, V^{\prime} \cap A_{j} \neq \varnothing$, $V \cap V^{\prime \prime} \neq \varnothing$, and $V^{\prime \prime} \cap A_{k} \neq \varnothing$. Then $V^{\prime}, V^{\prime \prime} \subset V^{*} \subset$ some element of $\mathscr{U}$. Since no element of $\mathscr{U}$ meets more than one member of $\left\{A_{j}\right\}$, it follows that $j=k$. Hence, every element of $\mathscr{U}$ meets at most one member of $\left\{V_{j}\right\}_{j \in J}$ so $\left\{V_{j}\right\}_{j \in J}$ is a discrete family of open neighborhoods of $\left\{A_{j}\right\}_{j \in J}$, respectively. If $U$ is any open 
neighborhood of $A=\bigcup A_{j}$, then $\left\{U \cap V_{j}\right\}_{j \in J}$ is a discrete family of open neighborhoods of $\left\{A_{j}\right\}$, respectively, contained in $U$. For each $j \in J$ let $N_{j}$ be a closed neighborhood of $A_{j}$ contained in $U \cap V_{j}$. Then $\left\{N_{j}\right\}$ is a discrete family of closed neighborhoods of $\left\{A_{j}\right\}$, respectively, whose union is contained in $U$. This implies that the collection of unions of discrete families $\left\{N_{j}\right\}$ where $N_{j}$ is a closed neighborhood of $A_{j}$ for each $j \in J$ is cofinal in the family of all closed neighborhoods of $A$ in $X$. Therefore,

$$
\begin{aligned}
\bar{H}(A) \approx & \underset{\lim }{\rightarrow}\left\{H(N) \mid N=\cup N_{j},\left\{N_{j}\right\}\right. \text { discrete, } \\
\left.\qquad N_{j} \text { a closed neighborhood of } A_{j} \text { in } X\right\} & \lim _{\rightarrow}\left\{\prod_{j \in J} H\left(N_{j}\right) \mid\left\{N_{j}\right\}\right. \text { discrete, } \\
\left.N_{j} \text { a closed neighborhood of } A_{j} \text { in } X\right\} & \\
\approx & \prod_{j \in J} \bar{H}\left(A_{j}\right)
\end{aligned}
$$

so $\bar{H}$ is additive.

Clearly there is a natural homomorphism $\phi: \bar{H}, \bar{\delta} \rightarrow H, \Delta$ defined by $\phi\{u\}=u \mid A$ for $u \in H(N), N$ a closed neighborhood of $A$. Then $\phi$ is a homomorphism between two additive $L$ theories on the paracompact space $X$. The hypothesis (1) above implies that $\phi_{x}$ is an isomorphism for every $x \in X$. By [5, Theorem 3.2] $\phi$ is an isomorphism of $L$ theories. Therefore, $H, \Delta$ is isomorphic to the $L$ theory determined by the cohomology theory $\bar{H}, \bar{\delta}$.

Cohomology theories on $X$ frequently arise from suitable contravariant functors defined on $\operatorname{cl}(X)^{2}$, the category of pairs of closed subsets of $X$. We formalize this using the following Eilenberg-Steenrod axioms. An ES theory $H, \delta^{*}$ on $X$ consists of:

(i) a contravariant functor $H$ from $\operatorname{cl}(X)^{2}$ to the category of graded abelian groups, and

(ii) a natural transformation of degree $1, \delta^{*}: H^{q}(B, \varnothing) \rightarrow H^{q+1}(A, B)$, for every $(A, B)$ in $\operatorname{cl}(X)^{2}$ such that the following hold:

Continuity. For every closed $A$ in $X$ there is an isomorphism

$$
\rho: \lim _{\rightarrow}\left\{H^{q}(N, \varnothing) \mid N \text { a closed neighborhood of } A \text { in } X\right\} \approx H^{q}(A, \varnothing)
$$

where $\rho\{u\}=u \mid(A, \varnothing)$ for $u \in H^{q}(N, \varnothing)$.

ExACTNESS. For every closed pair $(A, B)$ in $X$ the following sequence is exact

$$
\cdots \stackrel{\delta^{*}}{\rightarrow} H^{q}(A, B) \stackrel{H(j)}{\rightarrow} H^{q}(A, \varnothing) \stackrel{H(i)}{\rightarrow} H^{q}(B, \varnothing) \stackrel{\delta^{*}}{\rightarrow} H^{q+1}(A, B) \rightarrow \cdots
$$

where $i:(B, \varnothing) \subset(A, \varnothing)$ and $j:(A, \varnothing) \subset(A, B)$.

Excision. For closed sets $A, B$ in $X$ there is an isomorphism $\rho: H(A \cup B, B) \approx$ $H(A, A \cap B)$.

Thus, an ES theory satisfies some of the Eilenberg-Steenrod axioms [2] on $\operatorname{cl}(X)^{2}$. It need not satisfy the homotopy axiom nor the dimension axiom. 
Proposition (3.2). If $H, \delta^{*}$ is an ES theory on $X$, there is a cohomology theory $H^{\prime}$, $\delta^{\prime}$ on $X$ such that $H^{\prime}(A)=H(A, \varnothing)$ and $\delta^{\prime}: H^{\prime}(A \cap B) \rightarrow H^{\prime}(A \cup B)$ is suitably defined.

Proof. It is standard [2] that the exactness property of $H$ implies that $H^{\prime}(\varnothing)=$ $H(\varnothing, \varnothing)=0$ and the exactness and excision properties of $H, \delta^{*}$ imply the exactness of Mayer-Vietoris sequences with $\delta^{\prime}$ suitably defined. The continuity of $H^{\prime}$ follows from that of $H$.

In general we do not have a way of associating to a cohomology theory on $X$ an ES theory on $X$. With suitable definitions of cohomology theories and ES theories on larger categories it can be shown that the two theories are equivalent on the category of all compact spaces.

The concepts of nonnegativity, compactly supported and additivity are defined for ES theories to correspond to the same properties of the associated cohomology theories.

Most of the cohomology theories on $X$ we consider will be obtained from an ES theory on $X$ using Proposition (3.2).

Example (3.3). Define ${ }^{\Delta} H$ on $\operatorname{cl}(X)^{2}$ by ${ }^{\Delta} H^{q}(A, B)=H_{-q}(X-B, X-A)$ (singular homology with an arbitrary but fixed coefficient group) and define $\delta^{*}:{ }^{\Delta} H^{q}(B, \varnothing) \rightarrow{ }^{\Delta} H^{q+1}(A, B)$ to equal the connecting homomorphism d: $H_{-q}(X, X-B) \rightarrow H_{-q-1}(X-B, X-A)$ of the triple $(X, X-B, X-A)$. Exactness of the homology sequence of the triple $(X, X-B, X-A)$ yields exactness of ${ }^{\Delta} H, \delta^{*}$. The excision property for ${ }^{\Delta} H$ follows from the fact that $A, B$ closed in $X$ imply that $X-B, X-A$ are open in $X$ so the inclusion map

$$
(X-B,(X-A) \cap(X-B)) \subset((X-A) \cup(X-B), X-A)
$$

induces isomorphisms of singular homology.

The continuity property for ${ }^{\Delta} H$ follows from the fact that singular homology is carried by compact sets and the fact that, as $N$ varies over closed neighborhoods of $A$ in $X, X-N$ varies over a collection of open subsets of $X$ directed upward by inclusion whose union equals $X-A$ and this implies [8, Theorem 4.4.6]

$$
\stackrel{\lim }{\rightarrow}\left\{H_{-q}(X, X-N) \mid N \text { a closed neighborhood of } A \text { in } X\right\} \approx H_{-q}(X, X-A) \text {. }
$$

Therefore, $H, \delta^{*}$ is an ES theory on $X$.

In case $X$ is locally compact this theory is compactly supported. In fact, if $z \in$ ${ }^{\Delta} H^{q}(A)=H_{-q}(X, X-A)$ there is an open set $U$ with compact closure $\bar{U}$ such that $z$ is in the image of $H_{-q}(U \cup(X-A), X-A) \rightarrow H_{-q}(X, X \cup A)$. Then $A=$ $(A-U) \cup \overline{A \cap U}$ where $A-U=B$ is closed, $A \cap \bar{U}=C$ is compact and $z \mid B=$ 0 because $X-B=U \cup(X-A)$ so the composite

$$
H_{-q}(U \cup(X-A), X-A) \rightarrow H_{-q}(X, X-A) \rightarrow H_{-q}(X, X-B)
$$

is zero.

In Example (3.3) the same construction can be applied to other covariant functors in addition to singular homology as long as the functor has properties that yield the axioms for an ES theory (i.e. the functor defined on open pairs of $X$ should satisfy 
exactness and excision and should be compactly supported). The next example uses a limiting procedure similar to that in the proof of Theorem 3.1 to obtain a cohomology theory on a normal space $X$ from a suitable contravariant functor on open subsets of $X$ (see pp. 289-292 of [8] where the same procedure is applied to singular cohomology).

EXAMPLE (3.4). Let $H$ be a contravariant functor from the category of open subsets of $X$ to the category of graded abelian groups and let $\delta$ be a natural transformation $\delta: H^{q}(U \cap V) \rightarrow H^{q+1}(U \cup V)$ defined for two arbitrary open subsets $U, V \subset X$ such that $M V$ exactness holds for every open $U, V \subset X$. Define a contravariant functor $\bar{H}$ on the category of closed subsets of $X$ by

$$
\bar{H}^{q}(A)=\lim _{\rightarrow}\left\{H^{q}(U) \mid U \text { an open neighborhood of } A \text { in } X\right\}
$$

for every closed $A \subset X$. Clearly $\bar{H}$ is continuous on cl $X$. If $X$ is a normal space, as $U, V$ over open neighborhoods of $A, B$, respectively, $U \cup V$ and $U \cap V$ vary over cofinal families of open neighborhoods of $A \cup B$ and $A \cap B$, respectively. Thus, for a normal space $X$ there is a natural transformation $\bar{\delta}: \bar{H}^{q}(A \cap B) \rightarrow \bar{H}^{q+1}(A \cup B)$ for closed $A, B \subset X$ which is defined to be the direct limit of the natural transformations $\delta: H^{q}(U \cap V) \rightarrow H^{q+1}(U \cup V)$ where $U, V$ vary over open neighborhoods of $A, B$, respectively. Since the direct limit of exact sequences is exact, $\bar{H}, \bar{\delta}$ satisfy $M V$ exactness for closed $A, B \subset X$. In case $X$ is paracompact and $H$ is additive on discrete families of open subsets of $X$ it is easily verified (by an argument similar to that in the proof of additivity in the proof of Theorem (3.1)) that $\bar{H}$ is additive on $\operatorname{cl}(X)$.

4. Finite-dimensional spaces. First we present an example to show that the main uniqueness theorem [9, Theorem 2.20] generally fails if $H, H^{\prime}$ are not nonnegative cohomology theories on $X$. Then we prove that the uniqueness theorem is valid for arbitrary $H, H^{\prime}$ if $X$ is assumed to be a finite-dimensional space (finite-dimensional will mean a finite-dimensional separable metric space, as in [3]). We then present some applications of this uniqueness theorem to manifolds.

We consider the cohomology theory $K(X)$ defined for a compact Hausdorff space using complex vector bundles over $X$. It is well known [4] that $K$ is a cohomology theory on every compact Hausdorff space $X$ and that it is periodic of order 2 (i.e. $K^{q+2}(A) \approx K^{q}(A)$ for all $\left.q\right)$. Since $K^{0}(A) \approx \mathbf{Z} \oplus \tilde{K}(A)$, it follows that $K$ is not a nonnegative cohomology theory.

In [10] there is given an example ${ }^{2}$ of a continuous map $f: X \rightarrow Y$ between compact Hausdorff spaces such that for every $y \in Y, f \mid f^{-1} y: f^{-1} y \rightarrow y$ induces an isomorphism of $K(y)$ with $K\left(f^{-1} y\right)$ but $K(Y)$ is not isomorphic to $K(X)$. There is a homomorphism $f$ from the cohomology theory $K$ on $Y$ to the direct image (as in Example 2.6) $f_{*} K$ (which is also a cohomology theory on $Y$ ). This homomorphism is an isomorphism for every $y \in Y$ but is not an isomorphism for $Y$ itself. Thus, the uniqueness theorem of [9] is not true for arbitrary cohomology theories on a compact Hausdorff space.

\footnotetext{
${ }^{2}$ The author is indebted to $\mathrm{S}$. Ferry for pointing out this example to him.
} 
In order to extend the uniqueness theorem to arbitrary cohomology theories we need an inductive argument based on something other than degree of cohomology. Such an argument is possible based on dimension of the subset $A \subset X$. First we establish the following property of finite-dimensional spaces needed for the inductive proof.

Lemma (4.1). Let $A$ be a compact metric space of dimension q. Given $\varepsilon>0$ there exists a finite number of closed sets $A_{1}, \ldots, A_{m}$ such that

(1) $A=A_{1} \cup \cdots \cup A_{m}$,

(2) $\operatorname{diam} A_{i} \leqslant \varepsilon$ for $1 \leqslant i \leqslant m$,

(d) $\operatorname{dim}\left(A_{i} \cap A_{j}\right)<q$ for $1 \leqslant i \neq j \leqslant m$.

Proof. Because $A$ is a compact metric space of dimension $q$, there is a finite open covering $A=U_{1} \cup \cdots \cup U_{m}$ such that $\operatorname{diam} U_{i}<\varepsilon$ for $1 \leqslant i \leqslant m$ and dim boundary $U_{i}<q$ for $1 \leqslant i \leqslant q$.

Define $A_{i}=\bar{U}_{i}-\left(U_{1} \cup \cdots \cup U_{i-1}\right)$. Then $A_{1}, \ldots, A_{m}$ clearly satisfy (1) and (2) and, because $i<j$ implies $A_{i} \cap A_{j} \subset$ boundary $U_{i}$, (3) is also satisfied.

We now prove the uniqueness theorem for finite-dimensional spaces.

THEOREM (4.2). Let $\phi: H \rightarrow H^{\prime}$ be a homomorphism of cohomology theories on a metric space $X$ and let $n \in \mathbf{Z}$ be such that $\phi_{x}: H(x) \rightarrow H^{\prime}(x)$ is an n-equivalence for every $x \in X$. Then $\phi_{A}$ is an n-equivalence for every compact finite-dimensional subset $A \subset X$.

Proof. We prove the theorem by induction on the dimension of the subset $A$. If $A$ has dimension -1 , then $A=\varnothing$ and $\phi_{\varnothing}$ is an isomorphism because $H(\varnothing)=0=$ $H^{\prime}(\varnothing)$ so $\phi_{\varnothing}$ is an $n$-equivalence. Assume $A$ is compact, $\operatorname{dim} A=q>-1$ and the result is valid for all compact subsets of dimension $<q$.

(1) We prove $\phi_{A}: H^{k}(A) \rightarrow H^{\prime k}(A)$ is an epimorphism for $k<n$. Let $u \in H^{\prime k}(A)$, $k<n$, and assume $u \notin \operatorname{im} \phi_{A}$. By Lemma 4.1 we have closed sets $B_{1}, \ldots, B_{m}$ such that $A=B_{1} \cup \cdots \cup B_{m}$, diam $B_{i} \leqslant 1$ for $1 \leqslant i \leqslant m$ and $\operatorname{dim} B_{i} \cap B_{j}<q$ for $1 \leqslant i$ $\neq j \leqslant m$. We claim there is some $i$ with $1 \leqslant i \leqslant m$ such that $u \mid B_{i} \notin \operatorname{im} \phi$. In fact, if there is no such $i$ let $C_{j}=B_{1} \cup \cdots \cup B_{j}$ for $1 \leqslant j \leqslant m$. We prove by induction on $j$ that $u \mid C_{j} \in \operatorname{im} \phi$, which will give a contradiction because $C_{m}=A$ and $u \mid C_{m}=u$ $\notin \operatorname{im} \phi$ by hypothesis. For $j=1$ we know by hypothesis on $u \mid B_{i}$ that $u\left|C_{1}=u\right| B_{1}$ $\in \operatorname{im} \phi$. Assume $j>1$ and $u \mid C_{j-1} \in \operatorname{im} \phi$. The following diagram has exact rows and commutes up to sign

$$
\begin{array}{ccccccc}
H^{k-1}\left(C_{j-1} \cap B_{j}\right) & \stackrel{\delta}{\rightarrow} & H^{k}\left(C_{j}\right) & \stackrel{\alpha}{\rightarrow} & H^{k}\left(C_{j-1}\right) \oplus H^{k}\left(B_{j}\right) & \stackrel{\beta}{\rightarrow} & H^{k}\left(C_{j-1} \cap B_{j}\right) \\
\phi \downarrow \approx & & \phi \downarrow & & \phi \downarrow & & \approx \downarrow \phi \\
H^{\prime k-1}\left(C_{j-1} \cap B_{j}\right) & \stackrel{\delta^{\prime}}{\rightarrow} & H^{\prime k}\left(C_{j}\right) & \stackrel{\alpha^{\prime}}{\rightarrow} & H^{\prime k}\left(C_{j-1}\right) \oplus H^{\prime k}\left(B_{j}\right) & \stackrel{\beta^{\prime}}{\rightarrow} & H^{\prime k}\left(C_{j-1}^{k} \cap B_{j}\right)
\end{array}
$$

and the first and last vertical homomorphisms are isomorphisms because $k<n$ and

$$
\begin{aligned}
\operatorname{dim}\left(C_{j-1} \cap B_{j}\right) & \leqslant \operatorname{dim}\left(\left(B_{1} \cup \cdots \cup B_{j-1}\right) \cap B_{j}\right) \\
& \leqslant \operatorname{dim}\left(B_{1} \cap B_{j} \cup \cdots \cup B_{j-1} \cap B_{j}\right)<q .
\end{aligned}
$$


By [9, Lemma 2.19, part 2] $\alpha^{\prime-1}(\operatorname{im} \phi) \subset \operatorname{im} \phi$. The inductive hypothesis on $u \mid C_{j-1}$ implies $u \mid C_{j-1} \in \operatorname{im} \phi$. Also we have supposed $u \mid B_{j} \in \operatorname{im} \phi$. Therefore, $\alpha^{\prime}\left(u\left(C_{j}\right)\right) \in$ $\operatorname{im} \phi$ so $u \mid C_{j} \in \operatorname{im} \phi$. This completes the induction. Thus, the assumption that $u \mid B_{i} \in \operatorname{im} \phi$ for $1 \leqslant i \leqslant m$ leads to a contradiction so there is some $i$ such that $u \mid B_{i} \notin \operatorname{im} \phi$.

Choose $A_{1}$ to be a $B_{i}$ such that $u \mid A_{1} \notin \operatorname{im} \phi$. Then $A \supset A_{1}$, diam $A_{1} \leqslant 1$ and $u \mid A_{1} \notin \operatorname{im} \phi$. Repeat the argument to obtain $A_{1} \supset A_{2}, A_{2}$ closed, $\operatorname{diam} A_{2} \leqslant \frac{1}{2}$ and $u \mid A_{2} \notin$ im $\phi$. Continue to obtain a decreasing sequence of closed sets $A \supset A_{1} \supset A_{2}$ $\supset \cdots$ such that $\operatorname{diam} A_{i} \leqslant 1 / 2^{i-1}$ and $u \mid A_{i} \notin \operatorname{im} \phi$. Then $\cap A_{i}$ is a single point, say $x$, and by continuity of $H, H^{\prime}$, it follows that $u \mid x \notin \operatorname{im} \phi_{x}$ contradicting the hypothesis that $\phi_{x}$ is an epimorphism in dimensions $<n$. Therefore, $u \in \operatorname{im} \phi_{A}$ so $\phi_{A}$ is an epimorphism for dimensions $<n$.

(2) We prove $\phi_{A}: H^{k}(A) \rightarrow H^{\prime k}(A)$ is a monomorphism for $k \leqslant n$. Assume $u \in H^{k}(A), k \leqslant n$ is such that $\phi_{A}(u)=0$. We want to prove $u=0$. Assume $u \neq 0$ and let $A=B_{1} \cup \cdots \cup B_{m}$ be such that $B_{i}$ is closed and diam $B_{i} \leqslant 1$ for $1 \leqslant i \leqslant m$ and $\operatorname{dim}\left(B_{i} \cap B_{j}\right)<q$ for $1 \leqslant i \neq j \leqslant m$. We claim $u \mid B_{i} \neq 0$ for some $i$ with $1 \leqslant i \leqslant m$. Otherwise let $C_{j}=B_{1} \cup \cdots \cup B_{j-1}$ for $1 \leqslant j \leqslant m$. We prove by induction on $j$ that $u \mid C_{j}=0$ (this would give a contradiction because $0=u\left|C_{m}=u\right| A$ $=u \neq 0$ ). For $j=1$ we know by hypothesis on $u \mid B_{i}$ that $u\left|C_{1}=u\right| B_{1}=0$. Assume $j>1$ and that $u \mid C_{j-1}=0$.

The following diagram has exact rows and comutes up to sign

$$
\begin{array}{ccccccc}
H^{k-1}\left(C_{j-1}\right) \oplus H^{k-1}\left(B_{j}\right) & \stackrel{\beta}{\rightarrow} & H^{k-1}\left(C_{j-1} \cap B_{j}\right) & \stackrel{\delta}{\rightarrow} & H^{k}\left(C_{j}\right) & \stackrel{\alpha}{\rightarrow} & H^{k}\left(C_{j-1}\right) \oplus H^{k}\left(B_{j}\right) \\
\phi \downarrow & & \phi \downarrow \approx & & \downarrow \phi & & \downarrow \phi \\
H^{\prime k-1}\left(C_{j-1}\right) \oplus H^{\prime k-1}\left(B_{j}\right) & \stackrel{\beta^{\prime}}{\rightarrow} & H^{\prime k-1}\left(C_{j-1} \cap B_{j}\right) & \stackrel{\delta^{\prime}}{\rightarrow} & H^{\prime k}\left(C_{j}\right) & \stackrel{\alpha^{\prime}}{\rightarrow} & H^{\prime k}\left(C_{j-1}\right) \oplus H^{\prime k}\left(B_{j}\right)
\end{array}
$$

and the first vertical homomorphism is an epimorphism by (1) above and the second vertical homomorphism is an isomorphism because $\operatorname{dim}\left(C_{j-1} \cap B_{j}\right)<q$. By $[9$, Lemma 2.19, part 1], $\operatorname{ker} \alpha \cap \operatorname{ker} \phi=0$. Since

$$
\alpha\left(u \mid C_{j}\right)=\left(u\left|C_{j-1}, u\right| B_{j}\right)=(0,0)=0
$$

$u \mid C_{j} \in \operatorname{ker} \alpha \cap \operatorname{ker} \phi$ so $u \mid C_{j}=0$. This completes the induction. Thus, the hypothesis that $u \mid B_{i}=0$ for all $1 \leqslant i \leqslant m$ leads to a contradiction so there is some $i$ such that $u \mid B_{i} \neq 0$.

Choose $A_{1}$ to be a $B_{i}$ such that $u \mid A_{1} \neq 0$, continue as above to obtain a decreasing sequence of closed sets $A \supset A_{1} \supset A_{2} \supset \cdots$ such that diam $A_{i} \leqslant 1 / 2^{i-1}$ and $u \mid A_{i} \neq 0$. Then $\cap A_{i}$ is a point $x \in X$ and by continuity of $H$ and $H^{\prime}, u \mid x \neq 0$ contradicting the hypothesis that $\phi_{x}: H^{k}(x) \rightarrow H^{\prime k}(x)$ is a monomorphism for $k \leqslant n$. Therefore, $u=0$ so $\phi_{A}$ is a monomorphism for dimensions $\leqslant n$.

COROllaRY (4.3). Let $\phi: H \rightarrow H^{\prime}$ be a homomorphism of cohomology theories with compact supports on a finite-dimensional space $X$ and let $n$ be such that $\phi_{x}: H(x) \rightarrow$ $H^{\prime}(x)$ is an n-equivalence for every $x \in X$. Then $\phi_{A}$ is an n-equivalence for every closed $A \subset X$. 
Proof. By Theorem $4.2 \phi_{A}$ is an $n$-equivalence for every compact subset $A \subset X$. The corollary follows from this and Proposition (2.8).

Corollary (4.4). Let $H$ be a cohomology theory with compact supports on a finite-dimensional space such that $H(x)$ is nonnegative for every $x \in X$. Then $H(A)$ is nonnegative for all closed subsets $A \subset X$.

Proof. This follows from Corollary (4.3) using the obvious homomorphism from the trivial cohomology theory on $X$ to $H$.

EXAMPLES (4.5). Let $X$ be an $n$-manifold with or without boundary and consider the cohomology ${ }^{4} H$ defined in Example (3.3) on $X$. This is a cohomology theory on $X$ with compact supports such that ${ }^{\Delta} H^{q}(x)=H_{-q}(X, X-x)=0$ for $q \neq-n$ so $\sigma^{-n \Delta} H(x)$ is nonnegative for every $x \in X$ (where $\sigma^{-n \Delta} H$ is as defined in Remark (2.3)). By Corollary (4.4) $\sigma^{-n \Delta} H(A)$ is nonnegative for all closed $A$ or, equivalently, $H_{q}(X, X-A)=0$ for all $q>n$ and all closed $A \subset X$.

(4.6) Let $X$ be an $n$-manifold with boundary $\dot{X}$. We prove that for singular homology $H_{q}(X-\dot{X}) \approx H_{q}(X)$ for all $q$ (see also Lemma 11.7 of [6]). It suffices to prove $H_{q}(X, X-\dot{X})=0$ for all $q$. Let ${ }^{\Delta} H$ be the compactly supported cohomology theory on $X$ defined in Example (3.3) and consider its restriction $H^{\prime}$ to $\dot{X}$ (i.e. for $A$ closed in $\dot{X}$ we have $\left.H^{\prime q}(A)=H_{-q}(X, X-A)\right)$. Then $H^{\prime}(x)=0$ for all $x \in \dot{X}$. The unique homomorphism $\phi: 0 \rightarrow H^{\prime}$ of the trivial cohomology to $H^{\prime}$ is such that $\phi_{x}$ is an isomorphism for all $x \in \dot{X}$. By Corollary (4.3), $\phi_{A}$ is an isomorphism for all closed $A \subset \dot{X}$. Taking $A=\dot{X}$ we see that $H_{q}(X, X-\dot{X})=H^{\prime-q}(\dot{X})=0$ for all $q$.

Corollary (4.7). Let $\phi: H \rightarrow H^{\prime}$ be a homomorphism of additive cohomology theories on a locally compact finite-dimensional space $X$ such that, for some $n, \phi_{x}$ is an $n$-equivalence for every $x \in X$. Then $\phi_{A}$ is an n-equivalence for every closed $A \subset X$.

Proof. By Theorem (4.2), $\phi_{A}$ is an $n$-equivalence for every compact $A \subset X$. Since $X$ is locally compact, $X$ has an open covering $\mathscr{U}$ such that every closed subset contained in some element of $\mathscr{U}$ is compact. The corollary follows from this and Proposition (2.9).

EXAMPLE (4.8). Let $X$ be a paracompact $n$-manifold with boundary $\dot{X}$. We prove that for Cěch cohomology $\check{H}^{q}(X) \approx \check{H}^{q}(X-\dot{X})$ for all $q$ (see also Lemma 11.10 of [6]). It suffices to prove $\breve{H}^{q}(X, X-\dot{X})=0$ for all $q$. Let $H$ be the contravariant functor defined on relatively open subsets $U \subset \dot{X}$ by

$$
H^{q}(U)=\check{H}^{q}(U \cup(X-\dot{X}), X-\dot{X})
$$

and for relatively open $U, V \subset \dot{X}$ let $\delta: H^{q}(U \cap V) \rightarrow H^{q+1}(U \cup V)$ be the natural transformation of Cěch cohomology $\check{\delta}: \check{H}^{q}((U \cap V) \cup(X-\dot{X}), X-\dot{X}) \rightarrow$ $\check{H}^{q+1}((U \cup V) \cup(X-\dot{X}), X-\dot{X})$. Then $H, \delta$ satisfy $M V$ exactness for relatively open subsets $U, V \subset \dot{X}$ and additivity on discrete families of relatively open subsets in $\dot{X}$. The procedure of Example (3.4) yields an additive cohomology theory $\bar{H}, \bar{\delta}$ on $\dot{X}$ and it is easily seen that $\bar{H}^{q}(x)=0$ for all $x \in X$. Thus, the obvious homomorphism from the trivial cohomology theory on $\dot{X}$ to $\bar{H}$ is an isomorphism for all $x \in \dot{X}$. By Corollary (4.7) it is an isomorphism for all closed $A \subset \dot{X}$. In particular 
taking $A=\dot{X}$ we have

$$
0=\bar{H}^{q}(\dot{X})=\check{H}^{q}(X \cup(X-\dot{X}), X-\dot{X})=\check{H}^{q}(X, X-\dot{X})
$$

for all $q$.

The result in Example (4.8) and the one in Example (4.6) for a paracompact $n$-manifold also follow from the fact that for a paracompact $n$-manifold $X$ the inclusion map $X-\dot{X} \subset X$ is a homotopy equivalence.

\section{REFERENCES}

1. J. Dugundji, Topology, Allyn and Bacon, Boston, Mass., 1966.

2. S. Eilenberg and N. E. Steenrod, Foundations of algebraic topology, Princeton Univ. Press, Princeton, N. J., 1952.

3. W. Hurewicz and H. Wallman, Dimension theory, Princeton Univ. Press, Princeton, N. J., 1941.

4. M. Karoubi, K-theory, Springer-Verlag, New York, 1978.

5. J. D. Lawson, Comparison of taut cohomologies, Aequationes Math. 2 (1973), 201-209.

6. W. S. Massey, Homology and cohomology, Marcel Dekker, New York, 1978.

7. E. Michael, Local properties of topological spaces, Duke Math. J. 21 (1954), 163-171.

8. E. Spanier, Algebraic topology, Springer-Verlag, New York, 1982.

9. Cohomology isomorphisms, Contemporary Math. 12 (1982), 315-329.

10. J. T. Taylor, A counterexample in shape theory, Bull. Amer. Math. Soc. 81 (1975), 629-632.

Department of Mathematics, University of California at Berkeley, Berkeley, California 94720 